\title{
Validation d'une méthode de dosage de la cocaïne dans les produits de saisies par CLHP/UV BD
}

\section{Validation of a method for the quantitation of cocaine in seized materials by HPLC/UV DAD}

Gisèle FERRARI, Carine GARCIA, Daniel CHOPINEAUX, Martine PERRIN*

Institut de Recherche Criminelle de la Gendarmerie Nationale, 1, Boulevard Théophile Sueur 93111 ROSNY-SOUS-BOIS Cedex

* Auteur à qui adresser la correspondance : Martine PERRIN, Institut de Recherche de la Gendarmerie Nationale, 1, Boulevard Théophile Sueur - 93111 ROSNY-SOUS-BOIS Cedex - Tél : 0149355083 - Fax : 0149355027

(Reçu le 10 avril 2003 ; accepté le 18 septembre 2003)

\section{$R \mathscr{E} S U M E$}

La détermination et la quantification de la cocaïne dans les produits stupéfiants saisis par les services de la gendarmerie, des douanes ou de la police est essentielle sur le plan juridique. C'est pourquoi, la qualité des résultats rendus doit être avérée. Il est donc essentiel de travailler avec des protocoles validés. Étant donné qu'il n'existe aucune norme ou référentiel de validation consacrés à l'analyse des produits de saisie, un protocole interne a été élaboré à partir du guide de validation de la Société Française des Sciences et Techniques Pharmaceutiques. Celui-ci a servi lors de la validation de la méthode de dosage de la cocaïne, de la benzoylecgonine (principal produit de dégradation de la cocaïne) et de ses principaux produits de coupage. Cette procédure permet de vérifier : la spécificité, la linéarité, l'exactitude, la fidélité et les limites de quantification et de détection de la méthode. Ainsi, sur les huit molécules testées, six, dont la cocaine, présentent des résultats satisfaisants pour l'ensemble de ces paramètres. En revanche, deux molécules, la procaïne et la benzoylecgonine ont échoué à au moins un des tests cités précédemment. L'étude effectuée permet donc de valider la méthode de quantification de la cocaïne et de certains de ses produits de coupage.

MOTS-CL ÉS

Cocaïne, Quantification, Validation statistique.

\section{SUMMARY}

The identification and quantitation of cocaine in seized material by gendarmerie, customs or police is essential for any legal case. Because the results given must be rigorous, it is necessary to work with validated procedures. Considering there is no reference method to validate the analysis of illicit substances in seized material, an intern protocol was initiated, based on an adapted form of a validation guide written by the Société Française des Sciences et Techniques Pharmaceutiques. This one was used to validate the quantitation of cocaine,benzoylecgonine (principal product from cocaine degradation) and its main adulterants. This procedure helps verifying the specificity, linearity, precision, accuracy, limit of detection, limit of quantitation, of this method. Among the eight molecules tested, six (including cocaine) showed satisfactory results. But two of them, procaine and benzoylecgonine, failed in at least one of the previously mentioned tests. The study admits the validation of this quantitation method for cocaine and some adulterants.

KEY-WORDS

Cocaine, Quantitation, Statistic validation. 


\section{Introduction}

La cocaïne est un alcaloïde extrait de la feuille d'un arbuste appelé Erythroxylum coca qui possède des propriétés psychostimulantes dont l'usage peut donner lieu à une dépendance. Cette substance peut être sniffée sous forme de poudre (chlorhydrate de cocaïne), injectée après mise en solution ou encore fumée sous forme de crack ou de free-base.

La cocaïne, l'ecgonine et ses esters (dont la benzoylecgonine) figurent sur la liste des stupéfiants définie par l'arrêté modifié du 22 février 1990 du ministère de la santé et de la protection sociale ainsi que sur la liste couverte par la convention des stupéfiants de l'ONU (1961) et de Vienne (1971) (JO Nº63 du 14 mars 1991, décret $\mathrm{N}^{\circ}$ 91-271). A ce titre, leur trafic, leur détention et leur usage sont répréhensibles par la loi. Le département de toxicologie de l'Institut de Recherche Criminelle de la Gendarmerie effectue l'analyse qualitative et quantitative de substances stupéfiantes présentes dans les produits saisis par les services de police, de gendarmerie ou des douanes, dans un cadre juridique défini.

Un certain nombre de publications $(1,2,3,4)$ traite de la détection et de la quantification de la cocaïne et de ses métabolites par chromatographie liquide haute performance. Cependant, la plupart du temps, la matrice en présence, est une matrice biologique et généralement le matériel employé est différent de celui utilisé dans notre laboratoire. Ainsi, aucun des articles référencés ne traite du dosage de la cocaïne dans les poudres, par conséquent, il n'est jamais fait état des produits de coupage utilisés. De ce fait, une méthode de dosage de la cocaïne, de la benzoylecgonine et de ses principaux produits de coupage par chromatographie liquide haute performance (CLHP) couplée à un détecteur à barrette de diodes a été mise au point au laboratoire. Celle-ci répond à nos besoins car elle permet de séparer la cocaïne de la benzoylecgonine et de six produits de coupage, absorbants en UV et fréquemment utilisés par les trafiquants.

Compte tenu de l'enjeu des résultats rendus en police scientifique, une démarche qualité a été entreprise à l'Institut depuis quelques années. Ainsi, chacune des méthodes utilisées doit faire l'objet d'une validation permettant d'avoir confiance en la qualité et la fiabilité des résultats. Le dosage des stupéfiants dans les produits de saisies n'étant pratiqué que par un petit nombre de laboratoires, il est difficile de trouver une méthode de validation complète et adaptée à nos besoins. Pour pallier cette problématique, une procédure a été mise en place à partir de plusieurs procédures de validation $(5,6,7)$, dont le guide de validation analytique de la
SFSTP, mais en adaptant le protocole à nos contraintes. Celle-ci permet de valider les méthodes de quantification des produits stupéfiants dans les poudres par vérification des paramètres suivants : linéarité, exactitude, fidélité, spécificité et détermination des limites de détection et de quantification. En revanche, la robustesse de la méthode ne sera pas étudiée puisqu'elle n'est pas requise par la note explicative CEE III/844/87-FR FINAL d'août 1989 (8). De plus, la robustesse n'étant rien d'autre qu'une mesure de la reproductibilité des résultats obtenus dans des conditions opératoires normales de laboratoire à laboratoire et d'un analyste à l'autre (5), elle ne présente que peu d'intérêt dans notre cas. La procédure élaborée, a été mise en œuvre lors de la validation de la méthode de dosage de la cocaïne, de son produit de dégradation absorbant en UV (la benzoylecgonine) ainsi que certains produits de coupage, absorbant également dans l'UV, tels que : le paracétamol, la procaïne, la caféine, la chloroquine, la lidocaïne et la benzocaïne $(9,10)$.

\section{Matériels et méthodes}

\section{Réactifs :}

Les solvants utilisés sont tous de pureté analytique :

Acétonitrile (pour gradient en chromatographie en phase liquide) (VWR®) ;

Dihydrogénophosphate de potassium : $\mathrm{KH}_{2} \mathrm{PO}_{4}$ cristallisé qualité pour analyse (VWR®) ;

Acide acétique $100 \%$ (VWR®).

Les produits classés sur la liste des stupéfiants (cocaïne et benzoylecgonine) sont commandés chez Lipomed ${ }^{\circledR}$ après autorisation délivrée par l'AFSSAPS (Agence Française de Sécurité Sanitaire des Produits de Santé). Le certificat d'analyse de la cocaïne de référence COC156-HC, indique une pureté de 99,89\%. Le certificat d'analyse de la benzoylecgonine mono hydratée, de référence COC-204-HY-20, indique une pureté de $99,88 \%$.

Tous les autres réactifs sont achetés chez SigmaAldrich et sont également de pureté analytique.

\section{Appareillage :}

La séparation par chromatographie liquide haute performance est effectuée sur une colonne hypersil BDSC18 $(3 \mu \mathrm{m} \times 3 \mathrm{~mm} \times 125 \mathrm{~mm})$. Cette colonne est installée sur une chaîne analytique Hewlett Packard série 1050 couplée à un détecteur UV-visible à barrette de diodes (de marque identique).

\section{Méthode :}

La méthode utilisée a fait l'objet d'une optimisation préalable. 
L'étalon interne choisi est l'éthyl paraben mis en solution dans un mélange ternaire eau/acétonitrile/acide acétique $(70 / 26 / 4 \mathrm{v} / \mathrm{v} / \mathrm{v})$ à une concentration de 30 $\mathrm{mg} / \mathrm{l}$.

La phase mobile est constituée d'un mélange de tampon phosphate $(0,02 \mathrm{M}$ et $\mathrm{pH}=3,2)$ et d'acétonitrile. Le gradient appliqué est le suivant :

$$
\begin{aligned}
& \text { - } \mathrm{C}_{\mathrm{i}} \text { (acétonitrile) }=10 \% \\
& \text { - } \mathrm{C}_{\mathrm{f}} \text { (acétonitrile) }=55 \% \\
& \text { - } \mathrm{T}_{\mathrm{G}}=11 \mathrm{~min} \text { (soit } 4,5 \% / \mathrm{min} \text { ) }
\end{aligned}
$$

Le débit utilisé est le débit optimum pour une phase fixe greffée avec des particules de $3 \mu \mathrm{m}$ soit $0,5 \mathrm{ml} / \mathrm{min}$ et la colonne est thermostatée à $30^{\circ} \mathrm{C}$.

\section{Principe}

L'objectif de la validation d'une procédure analytique est de démontrer qu'elle est appropriée à l'usage auquel elle est destinée. Chacun des paramètres exposés à la figure 1 doit être vérifié par un calcul afin d'obtenir une exploitation statistique pour l'ensemble des

\begin{tabular}{|c|c|}
\hline \multirow{6}{*}{ Linéarité } & Validité du coefficient de corrélation \\
\hline & Test de comparaison de l'ordonnée à l'origine avec 0 \\
\hline & Test de comparaison des pentes et des ordonnées à l'origine avec 0 \\
\hline & Test d'homogénéité des variances : test de Cochran \\
\hline & Test de l'existence d'une pente significative \\
\hline & Test de validité de la droite de régression \\
\hline \multirow{3}{*}{ Exactitude } & Test d'homogéneité des variances test de Cochran \\
\hline & Test de validité des moyennes \\
\hline & Calcul du recouvrement moyen et des intervalles de recouvrement \\
\hline \multirow{2}{*}{ Fidélité } & Test d'homogéneité des variances : test de Cochran \\
\hline & Etude des variances de répétabilité, intergroupe et de reproductibilité \\
\hline \multirow{2}{*}{ Spécificité } & Etude de la résolution et de la spécificité des pics \\
\hline & Vérification de l'interaction des composés les uns sur les autres \\
\hline Limites de détection & Limites de détection \\
\hline Limites de quantification & Vérification des critères de fidélité et d'exactitude \\
\hline
\end{tabular}
critères de performance. Les résultats obtenus nous permettent de déterminer si l'objectif visé est atteint.

Figure 1 : Principe de la validation statistique.

\section{Étude de la spécificité}

La spécificité est la capacité de la méthode à déterminer sans équivoque, l'analyte en présence. Elle garantit que le signal mesuré provient uniquement de la substance à analyser ou permet de mettre en évidence les interactions entre les molécules présentes de façon concomitante dans l'échantillon.

Dans le cas d'une poudre, il sera possible de conclure que la séparation est optimale si les valeurs des paramètres de séparation sont les suivantes : 1,5 pour le facteur de résolution Rs et 1,1 pour la sélectivité $\alpha$. Seules ces deux grandeurs sont prises en compte en ce qui concerne l'identification, puisque la matrice n'induit aucune interaction (matrice reconstituée) et que la pureté des produits utilisés est connue. Ces deux paramètres étant effectifs pour notre méthode (voir figure 2 et tableau I) celle-ci est dite spécifique sur le plan de l'identification.

En ce qui concerne la quantification, la spécificité de la méthode consiste à s'assurer que la molécule présente des caractéristiques chromatographiques identiques qu'elle soit seule ou en présence d'autres analytes. Elle est estimée lors de la comparaison des droites obtenues avec la matrice reconstituée et celles obtenues pour le produit pur (cf § "Étude de la linéarité et de l'exactitude"). Ceci tend à prouver qu'il n'y a pas d'interaction entre le milieu et le produit à quantifier.

\section{Étude de la linéarité et de l'exactitude}

\section{Linéarité}

La linéarité d'une procédure d'analyse est sa capacité, à l'intérieur d'un certain intervalle, à fournir des résultats directement proportionnels à la concentration en analyte dans l'échantillon. Cette proportionnalité s'exprime au travers d'une expression mathématique définie à priori. Le modèle étudié ici est un modèle linéaire d'équation $\mathrm{y}=\mathrm{bx}+\mathrm{a}$.

Les limites de linéarité sont les limites expérimentales des valeurs entre lesquelles un modèle d'étalonnage linéaire peut être appliqué avec un niveau de confiance connu (généralement pris égal à $1 \%$ ).

Le protocole d'étude employé pour étudier la linéarité se déroule en deux étapes :

- La première consiste à préparer cinq séries (i de 1 à $n$, avec $n=5$ ) de cinq ( $j$ de 1 à $p$, avec $p=5$ ) niveaux de concentration $(\mathrm{Q} 1$ à $\mathrm{Q} 5)$ dans la solution acide contenant l'étalon interne. Les solutions sont préparées à partir des poudres de pureté connue et doivent être refaites pour chacune des séries sans procéder par dilutions successives. Cette opération a pour objectif d'estimer la linéarité de chaque analyte dans une matrice équivalente à celles des produits saisis.

Les valeurs de $\mathrm{Q} 1$ à $\mathrm{Q} 5$ sont respectivement 10 - 20 30 - 40 et $50 \mathrm{mg} / \mathrm{l}$ pour la cocaïne et les produits de 


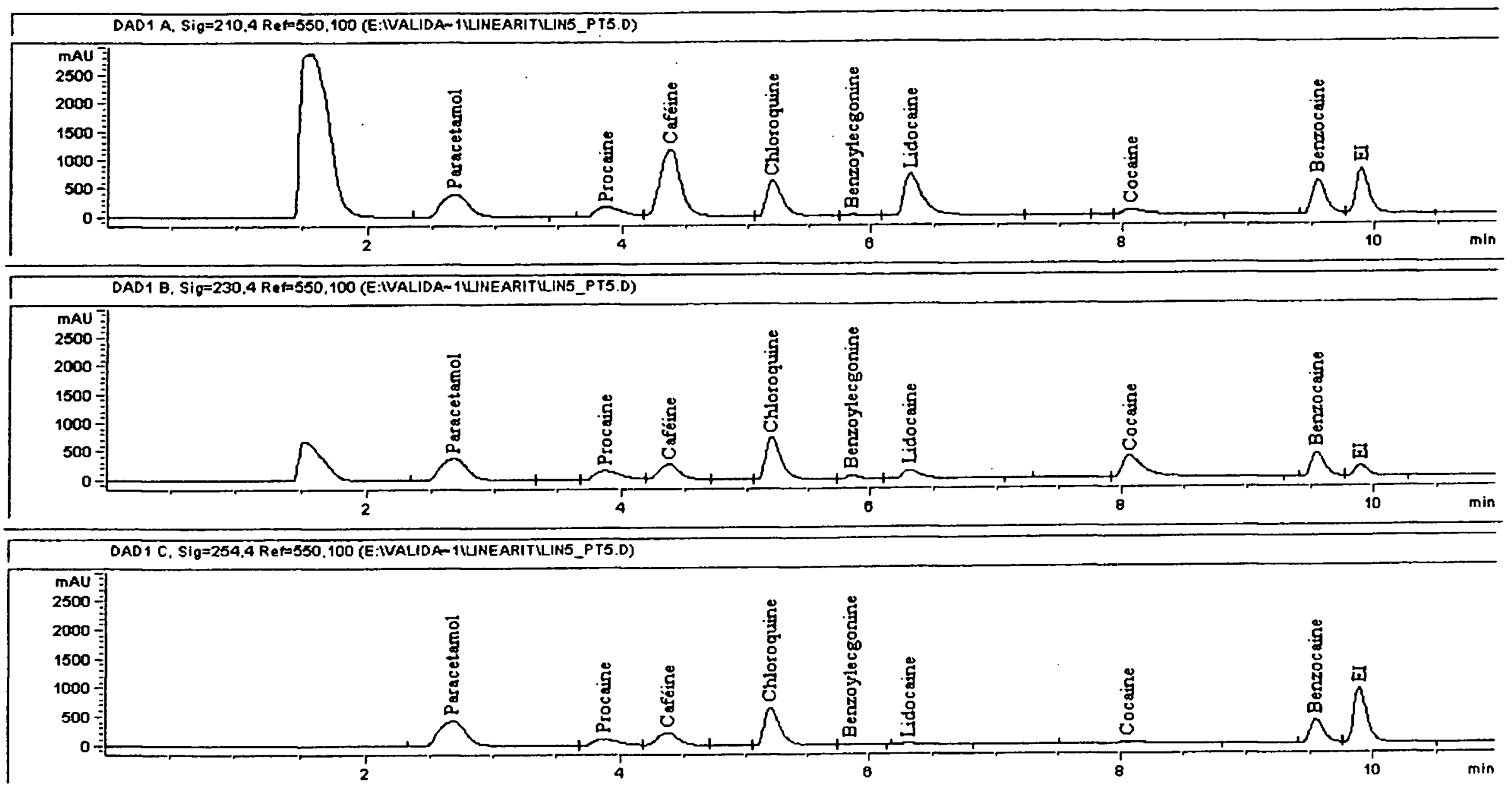

Figure 2 : Chromatogramme du point 5 (cocaïne à $\left.50 \mathrm{mg} . \mathrm{l}^{\prime \prime}\right)$, aux différentes longueurs d'onde, réalisé lors de l'étude de linéarité pour le mélange.

Tableau I: Valeurs des facteurs de résolution après optimisation de la méthode.

\begin{tabular}{|l|c|c|}
\hline & Valeurs de la sélectivité & Valeurs des facteurs de résolution \\
\hline Pic d'injection et Paracétamol & - & 3,14 \\
\hline Paracétamol et Procaïne & 2,04 & 3,01 \\
\hline Procaïne et Caféine & 1,21 & 1,51 \\
\hline Caféine et Chloroquine & 1,28 & 3,15 \\
\hline Chloroquine et Benzoylecgonine & 1,18 & 3,46 \\
\hline Benzoylecgonine et Lidocaïne & 1,11 & 2,32 \\
\hline Lidocaïne et Cocaïne & 1,37 & 6,86 \\
\hline Cocaïne et Benzocaïne & 1,23 & 6,54 \\
\hline Benzocaïne et Ethyl paraben & 1,11 & 1,89 \\
\hline
\end{tabular}

coupage, alors qu'elles sont de 1 - 2 - 3 - 4 et $5 \mathrm{mg} / \mathrm{l}$ pour la benzoylecgonine.

Les cinq gammes sont préparées et injectées en CLHP couplée à un détecteur à barrette de diodes. Les droites d'étalonnage sont tracées pour chacune des gammes et pour les molécules d'intérêts, à trois ou quatre longueurs d'onde différentes $(210 \mathrm{~nm}, 230 \mathrm{~nm}, 254 \mathrm{~nm}$ et $292 \mathrm{~nm}$ ) et pour l'étalon interne à $254 \mathrm{~nm}$. Celles-ci correspondent au maximum d'absorption des produits analysés. Cette validation sur plusieurs longueurs d'onde offrira une plus grande souplesse de travail.

La droite obtenue (D1) avec ces valeurs a pour équation : $y=b_{1} x+a_{1}$ soit $y=0,01431 x-0,00668$.
- La deuxième étape consiste à préparer cinq gammes ne contenant que l'analyte à considérer. La solution étalon sera de nouveau préparée pour chacune des répétitions. Les niveaux de concentration testés sont identiques aux précédentes (Q1 à Q5). La droite obtenue (D2) avec ces valeurs a pour équation : $y=b_{2} x+a_{2}$ soit $\mathrm{y}=0,01476 \mathrm{x}-0,00252$.

Étant donné que les deux analyses sont faites à partir des même produits, de pureté assimilée à $100 \%$, les écarts potentiellement obtenus ne pourront incomber qu'à des interactions entre les différentes molécules.

Les droites obtenues par ces deux procédés, seronı 
comparées afin de vérifier si les analytes possèdent le même comportement seuls et dans le mélange. Cependant, avant de procéder à ces comparaisons, les cinq gammes injectées pour le produit pur et pour le produit dans le mélange, doivent satisfaire les paramètres présentés ci-après.

Le tableau II, présente les résultats obtenus pour la cocaïne à $230 \mathrm{~nm}$, pour l'ensemble des tests réalisés lors de la linéarité.

\section{Coefficient de corrélation}

Le coefficient de corrélation $\left(\mathrm{r}^{2}\right)$, quand il est égal à 1 , indique l'existence d'une liaison linéaire entre $x$ et $y$. En revanche, s'il est inférieur à 1 , sa valeur nous renseigne sur l'intensité de cette liaison et doit être comparée à une valeur statistique pour juger de sa validité (11). D'après la table de distribution du coefficient de corrélation, pour cinq points de gamme et un seuil de probabilité de $1 \%$, la valeur du coefficient de corrélation doit être supérieure à 0,959 (voir la figure 3 ).

Ces coefficients sont corrects pour la plupart des longueurs d'onde testées, exceptée pour la benzoylecgonine à 210 et $254 \mathrm{~nm}$ où ces valeurs sont inférieures au seuil fixé.

Test de comparaison de l'ordonnée à l'origine avec 0

L'inégalité suivante va permettre d'établir la corrélation de notre droite avec zéro :

Avec :

$$
\frac{|\mathrm{a}|}{\mathrm{Sa}}<\mathrm{t}(\alpha ; \mathrm{N}-2)
$$

S(a) = écart type sur l'ordonnée à l'origine $\alpha=1 \%$ ( $\mathrm{t}$ de Student $(12,13)$ pour un test bilatéral avec un intervalle de confiance de $1 \%$ )

et $\mathrm{N}=25$ et $\mathrm{N}-2=23$

soit $\mathbf{t}(\mathbf{0 , 0 1} ; \mathbf{2 3})=\mathbf{2 , 8 0 7}$

Toutes les molécules passent ce test avec succès puisque les valeurs de $|\mathrm{a}| / \mathrm{Sa}$ sont toutes inférieures à 2,807 .

\section{Comparaison des pentes et des ordonnées à l'origine}

Le but de ce test est de vérifier qu'il n'y ait pas d'incohérence entre la droite moyenne obtenue pour chacun des produits mis en solution dans la matrice reconstituée et la droite moyenne de chaque produit pur.

\section{Comparaison des pentes des droites :}

Calcul de la statistique $t$ :

$$
t_{\text {test }}=\left|b_{1}-b_{2}\right| / \sqrt{S^{2} b_{1}+S^{2} b_{2}}
$$

Avec $\mathrm{t}<\mathrm{t}_{\text {test }}\left(\alpha ; \mathrm{N}_{1}+\mathrm{N}_{2}-4\right)$ lu dans la table de Student, soit $t_{\text {test }}<2,678$ pour que les pentes ne soient pas significativement différentes au risque d'erreur $\alpha$ de $1 \%$. Les valeurs de $b_{1}$ et de $b_{2}$ correspondent aux pentes des deux droites (Rappel : $\mathrm{N}_{1}$ : nombre de mesures pour la $1^{\text {ire }}$ série et $\mathrm{N}_{2}$ : nombre de mesures pour la $2^{\text {emc }}$ ).

Ces comparaisons invalident un certain nombre de longueurs d'onde qui dépassent la valeur critique de $t_{\text {test }}$. Ainsi, la procaïne à 210,230 et $254 \mathrm{~nm}$ et le paracétamol à 210 et $230 \mathrm{~nm}$ ne peuvent être acceptés.

\section{Comparaison des ordonnées à l'origine}

Calcul de la statistique $\mathrm{t}$ :

$$
\mathrm{t}_{\text {test }}=\left|\mathrm{a}_{1}-\mathrm{a}_{2}\right| / \sqrt{\mathrm{S}^{2} \mathrm{a}_{1}+\mathrm{S}^{2} \mathrm{a}_{2}}
$$

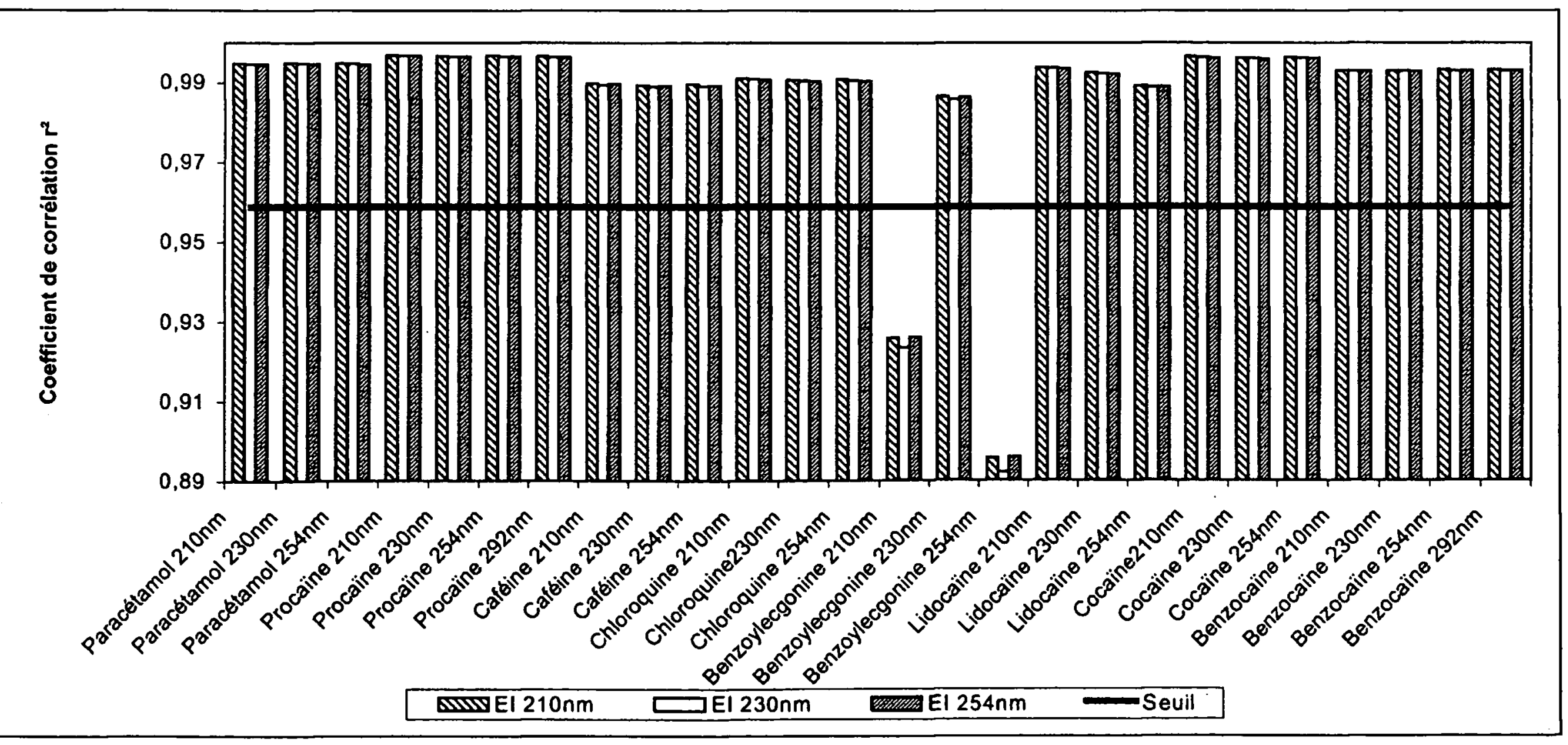

Figure 3 : Coefficient de corrélation des produits aux longueurs d'onde étudiées. 
Tableau II : Résultats obtenus pour les droites étalons de la cocaïne à $230 \mathrm{~nm}$ lors de l'étude de la linéarité dans le mélange (D1) et pour l'analyte seul (D2).

\begin{tabular}{|c|c|c|c|}
\hline & D1 & D2 & Seuils théorique au risque $1 \%$ \\
\hline (1) coefficient de corrélation & 0,996 & 0,999 & \\
\hline $\begin{array}{l}\text { (2) Test de comparaison des ordonnées à l'origine } \\
\text { avec } 0 \text { (test } t \text { ) }\end{array}$ & $\begin{array}{l}0,75 \\
(\mathrm{NS})\end{array}$ & $\begin{array}{l}0,47 \\
\text { (NS) }\end{array}$ & $\mathrm{t}(0,01 ; 23)=2,807$ \\
\hline (3) Test d'homogénéité des variances (Cochran) & $\begin{array}{l}0,4705 \\
\text { (NS) }\end{array}$ & $\begin{array}{l}0,349 \\
\text { (NS) }\end{array}$ & $\mathrm{C}(0,05 ; 5 ; 4)=0,544$ \\
\hline (4) Test de l'existence des pentes (Fisher) & $\begin{array}{l}2831 \\
\text { (HS) }\end{array}$ & $\begin{array}{l}8311 \\
\text { (HS) }\end{array}$ & $\mathrm{F}(0,01 ; 1 ; 23)=7,88$ \\
\hline (5) Validité des ajustements (Fisher) & $\begin{array}{c}0,149 \\
(\mathrm{NS})\end{array}$ & $\begin{array}{r}0,369 \\
\text { (NS) }\end{array}$ & $\mathrm{F}(0,01 ; 3 ; 20)=4,94$ \\
\hline $\begin{array}{l}\text { (6) Test de comparaison des ordonnées à l'origine } \\
\text { des droites d'ajustement (test } t \text { ) }\end{array}$ & \multicolumn{2}{|c|}{$\begin{array}{l}0,399 \\
(\mathrm{NS})\end{array}$} & $\mathrm{t}(0,05 ; 23)=2,678$ \\
\hline $\begin{array}{l}\text { (7) Test de comparaison des pentes des droites } \\
\text { d'ajustement (test } t \text { ) }\end{array}$ & \multicolumn{2}{|c|}{$\begin{array}{l}1,432 \\
(\mathrm{NS})\end{array}$} & $\mathrm{t}(0,05 ; 23)=2,678$ \\
\hline
\end{tabular}

NS : valeur non significative au risque $\alpha$

HS : valeur hautement significative au risque $<0,1 \%$

Les valeurs calculées doivent être inférieure aux valeurs théoriques sauf pour le test (4)

Avec $\mathrm{t}<\mathrm{t}_{\text {test }}\left(\alpha ; \mathrm{N}_{1}+\mathrm{N}_{2}-4\right)$ lu dans la table de Student, soit $t_{\text {test }}<2,678$ pour que les ordonnées à l'origine ne soient pas significativement différentes au risque d'erreur $\alpha$ de $1 \%$. Les valeurs de $a_{1}$ et de $a_{2}$ correspondent aux ordonnées à l'origine des deux droites.

Cette comparaison n'invalide aucune des molécules testées.

\section{Test d'homogénéité des variances ou test de Cochran} (5)

L'objectif de ce test est de vérifier l'homogénéité des variances constitutives de l'erreur expérimentale et ainsi de détecter la présence de valeurs suspectes.

Le critère à utiliser est :

$$
C_{\text {test }}=\frac{S^{2} \max }{\sum_{j=1}^{p} S_{j}^{2}}
$$

Avec :

$\mathrm{S}^{2} \max$ : Variance la plus élevée des groupes $\mathrm{j}$

$S_{j}^{2}$ : Variance du groupe $\mathrm{j}$ (sur 5 valeurs)

Si l'inégalité suivante est vérifiée pour $\mathrm{C}_{\text {test }}<\mathrm{C}(\alpha ; \mathrm{p} ; \mathrm{n}$ 1) avec $\mathrm{p}=5$ et $\mathrm{n}=5$ avec $\alpha=1 \%$ soit $\mathrm{C}_{\text {aberrant }}<0,633$ et $\alpha=5 \%$ soit $\mathrm{C}_{\text {crit }}<0,544$ (d'après la table de Cochran (13)). (Nota : $\mathrm{C}_{\text {crit }}=\mathrm{C}_{\text {critique }}$ )

L'ensemble des variances peut être considéré comme homogène lorsque $\mathrm{C}_{\text {test }}<\mathrm{C}_{\text {crit }}$. En revanche, si $\mathrm{C}_{\text {test }}$ est compris entre les valeurs critiques de la table aux seuils de 1 et $5 \%\left(\mathrm{C}_{\mathrm{aberrant}}\right.$ et $\left.\mathrm{C}_{\mathrm{cri}}\right)$, la variance la plus élevée est dite suspecte. Dans ce dernier cas, un test de Dixon devrait être pratiqué afin d'éliminer cette dernière.

Pour les huit mølécules testées, certaines valeurs de $\mathrm{C}_{\text {test }}$ sont comprises entre les deux seuils. Elles sont donc considérées comme suspectes. Ainsi, pour éliminer la présomption de présence de valeurs aberrantes, il faudrait utiliser le test de Dixon cité précédemment. Néanmoins, les analyses étant effectuées sur plusieurs longueurs d'onde pour chaque molécule, nous prendrons le parti de supprimer la totalité de la série douteuse en choisissant le seuil de validité le plus restrictif soit $95 \%\left(\mathrm{C}_{\text {crit }}\right)$. De ce fait, en ce qui concerne les molécules analysées seules en solution, la benzoylecgonine à $230 \mathrm{~nm}$ présente une valeur de $\mathrm{C}>\mathrm{C}_{\text {crit }}$ et cette longueur d'onde ne sera pas retenue.

Réaliser un test de l'existence d'une pente significative L'objectif est de comparer les variations dues à la régression et celles dues aux erreurs expérimentales et aux erreurs d'ajustement. Pour cela, il faut prouver que la pente de la droite, n'est pas seulement due à la variance résiduelle, mais que la droite possède une pente qui lui est propre (variance de régression > variance résiduelle).

Un certain nombre de sources de variation sont à prendre en compte :

- variance due à la régression linéaire : $\mathrm{S}_{\mathrm{i}}{ }^{2}$; 
- variance résiduelle $S_{R}{ }^{2}$, qui représente la différence entre la valeur théorique et la valeur réelle ;

- variance totale : $S_{T^{2}}$.

La vérification de l'existence d'une pente significative est établie à l'aide du test de Fisher $F_{1}$ en montrant que les variances $S_{i}{ }^{2}$ et $S_{R}{ }^{2}$ sont significativement différentes au risque $\alpha$ choisi (1\%):

$$
F_{1}=\frac{s_{\cdot l}^{2}}{s_{R}^{2}}
$$

La table de Fisher (12) donne : $\mathrm{F}_{\text {table }}(\alpha ; 1 ; \mathrm{N}-2)=\mathbf{7 , 8 8}$ (avec $\alpha=0,01, N=25$ et $1=$ variation due à la régression). Pour qu'il soit possible de conclure sur l'existence d'une pente significative, au seuil de probabilité considéré $\alpha=1 \%$, il faut que $F_{1}$ soit supérieur à 7,88 .

Les résultats obtenus pour ce test sont concluants. Ainsi, la totalité des droites étalons obtenues, possèdent une pente significative.

\section{Test de validité de la droite de régression}

L'objectif de ce test est de vérifier que les variances $S_{L}{ }^{2}$ et $S_{E}{ }^{2}$ ne sont pas significativement différentes au seuil de risque $\alpha$ choisi (1\%) :

- variance de l'erreur de régression $\mathrm{S}_{\mathrm{L}}{ }^{2}$, erreur du modèle ;

- variance de l'erreur expérimentale $\mathrm{S}_{\mathrm{E}}{ }^{2}$ (variation à l'intérieur de chaque niveau).

La validité de la droite de régression est représentée par $\mathrm{F}_{2}$ :

$$
F_{2}=\frac{S_{L}^{2}}{S_{E}^{2}}
$$

Avec $\mathrm{F}_{2}(\alpha ; \mathrm{k}-2 ; \mathrm{N}-\mathrm{k})=4,94$ (avec $\alpha=0,01, \mathrm{~N}=25$ et $\mathrm{k}=5$ ). La droite de régression est dite "valide", au seuil de probabilité choisi $\alpha=1 \%$, lorsque $F_{2}$ est inférieur à 4,94 .

Ainsi, la validité de toutes les droites est mise en évidence par ces calculs.

\section{Conclusion sur la linéarité des gammes}

L'ensemble de l'étude de la linéarité nous permet de conclure sur la validité de ce paramètre pour toutes les gammes. Les produits ayant échoué à l'un des tests sont considérés comme non linéaires. La suite de la validation statistique ne tiendra plus compte des longueurs d'onde invalidées lors des tests précédents. Sont considérés comme linéaires le paracétamol à $254 \mathrm{~nm}$, ainsi que la cocaïne, la caféine, la benzoylecgonine, la lidocaïne, la chloroquine et la benzocaïne à 210,230 et $254 \mathrm{~nm}$ (étalon interne mesuré à $254 \mathrm{~nm}$ ).

\section{Étude de l'exactitude}

L'exactitude d'une procédure analytique exprime l'étroitesse de l'accord entre la valeur de concentration acceptée comme référence ou la valeur vraie et la valeur déterminée par la procédure analytique.

Ce test vérifie si la variance des recouvrements obtenue à un niveau de concentration (variance intragroupe) est similaire à la variance des recouvrements moyens des différents niveaux de concentration (variance intergroupe). Ceci est le pré-requis pour que les données soient représentatives et permettent de réaliser le test de la valeur de recouvrement $100 \%$ dans l'intervalle de confiance considéré.

Vérification de l'homogénéité des variances liées ou test de Cochran

Comme pour la linéarité, ce test permet de déterminer la présence de valeurs suspectes. La formule du test de Cochran est utilisée comme précédemment et un exemple de calcul est présenté par le tableau III.

La benzocaïne à $254 \mathrm{~nm}$ présente une valeur de $\mathrm{C}_{\text {test }}$ supérieure au seuil de tolérance indiquant une probabilité de présence de valeurs aberrantes. Cette longueur d'onde est exclue pour la suite des tests.

Test de validité des moyennes :

- La variation totale $\left(\mathrm{S}_{\mathrm{T}}^{2}\right)$;

- La variation intra-groupe $\left(\mathrm{S}_{\mathrm{E}}^{2}\right)$;

- La variation intergroupe $\left(\mathrm{S}_{\mathrm{C}}^{2}\right)$.

L'ensemble de ces variances permet de calculer F3 qui illustre la cohérence entre la variance inter-groupe et la variance intra-groupe :

$$
\text { F3 calculé : } \quad F_{3}=\frac{S_{C}^{2}}{S_{E}^{2}}
$$

Ce test compare les erreurs intra-groupe et inter-groupe : si F3 $<\mathrm{F}(\alpha, \mathrm{p}-1, \mathrm{~N}-\mathrm{p})$ avec $\mathrm{p}=5, \mathrm{n}=5$ et $\mathrm{N}=25$ ou $\mathrm{F}=4,43$; alors il est possible de dire au risque $\alpha=$ $1 \%$ que les variations des recouvrements moyens aux différents niveaux sont seulement dues à l'erreur expérimentale.

Tous les produits passent ce test avec succès puisqu'ils ont tous un F3 inférieur au seuil fixé (exemple de calcul au tableau IV).

Tableau III : Test d'homogénéité des variances (test de Cochran) - Cocaïne $230 \mathrm{~nm}$.

\begin{tabular}{|c|c|c|c|c|c|}
\hline Groupes (j) & 1 & 2 & 3 & 4 & 5 \\
\hline $\mathrm{n}_{\mathrm{j}}$ & 5 & 5 & 5 & 5 & 5 \\
\hline $\mathrm{S}_{\mathrm{j}}$ & 4,1 & 6,72 & 9,52 & 6,05 & 4,57 \\
\hline
\end{tabular}
$\mathrm{C}_{\text {calculé }}=9,52 \times 30,96=0,3075<0,544$ (seuil le plus restrictif : $95 \%$ ).


Annales de Toxicologie Analytique, vol. XV, n 3, 2003

Tableau IV : Test de validité des moyennes - Cocaïne $230 \mathrm{~nm}$.

\begin{tabular}{|c|c|c|c|c|}
\hline 1) & FIDIS & Somine des a arres & Yaranes & 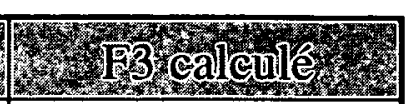 \\
\hline Variation totale & 24 & 134,64 & 5,6100 & \\
\hline Variation inter-groupes & 4 & 10,80 & 2,7012 & 0,4363 \\
\hline Variation intra-groupe & 20 & 123,84 & 6,1918 & \\
\hline
\end{tabular}

F3th $>4,43$

$\mathrm{F} 3=0,436<\mathrm{F}(0,01 ; 4 ; 20)=4,43$. la valeur de F3 n'est donc pas significative au risque $\alpha=1 \%$

\section{Estimation du recouvrement moyen}

Il est possible de déterminer le recouvrement moyen (RM) ainsi que son intervalle de confiance :

$$
R M=\sum_{j=1}^{k}\left(1 / k_{n j}\right) \sum_{i=1}^{n j} Y_{i j}
$$

avec $\mathrm{k}=$ nombre de groupes.

$$
I_{R M}=R M \pm \frac{t_{(\alpha ; N-1)} \cdot S_{T}}{\sqrt{N}}
$$

Avec $\mathrm{t}(\alpha ; \mathrm{N}-1)$ lu dans la table de Student pour $\mathrm{N}=25$ et $\alpha=0,01$ soit $\mathrm{t}=2,797$.

Les résultats sont présentés au tableau $\mathrm{V}$ et ils sont jugés satisfaisants.

\section{Étude de la fidélité}

\section{Définition}

La fidélité de la méthode exprime l'étroitesse de l'accord entre une série de mesures provenant de prises multiples d'un échantillon homogène, dans des conditions prescrites.

\section{Principe et protocole d'étude}

Les essais réalisés sur des produits identiques et dans des circonstances présumées identiques ne conduisent en général pas aux mêmes résultats. Ceci s'explique par l'existence d'erreurs à caractère aléatoire. Les facteurs qui peuvent contribuer à la variabilité d'une méthode d'essai sont nombreux et ne peuvent être tous rigoureusement contrôlés. L'étude de la fidélité permet d'approcher la variabilité entre des essais répétés.

La fidélité s'exprime par la mesure de la répétabilité

Tableau $V:$ Recouvrement moyen et indice de recouvrement des longueurs d'onde valides.

\begin{tabular}{|l|c|c|}
\hline & Recouvrement moyen (\%) & Intervalle de recouvrement (\%) \\
\hline Paracétamol à $254 \mathrm{~nm}$ & 99,95 & $\pm 0,86$ \\
\hline Caféine à $210 \mathrm{~nm}$ & 99,89 & $\pm 1,03$ \\
\hline Caféine à $230 \mathrm{~nm}$ & 100,07 & $\pm 1,05$ \\
\hline Caféine à $254 \mathrm{~nm}$ & 99,99 & $\pm 0,97$ \\
\hline Chloroquine à $210 \mathrm{~nm}$ & 100,05 & $\pm 1,12$ \\
\hline Chloroquine à $230 \mathrm{~nm}$ & 100,10 & $\pm 1,14$ \\
\hline Chloroquine à $254 \mathrm{~nm}$ & 100,11 & $\pm 1,12$ \\
\hline Lidocaïne à $210 \mathrm{~nm}$ & 99,88 & $\pm 1,03$ \\
\hline Lidocaïne à $230 \mathrm{~nm}$ & 100,04 & $\pm 1,09$ \\
\hline Lidocaïne à $254 \mathrm{~nm}$ & 100,10 & $\pm 1,10$ \\
\hline Cocaïne à 210 nm & 99,95 & $\pm 1,10$ \\
\hline Cocaïne à 230 nm & 99,97 & $\pm 1,32$ \\
\hline Cocaïne à 254 nm & 99,96 & $\pm 1,31$ \\
\hline Benzocaïne à $210 \mathrm{~nm}$ & 100,02 & $\pm 0,39$ \\
\hline Benzocaïne à $230 \mathrm{~nm}$ & 99,97 & $\pm 0,46$ \\
\hline Benzocaïne à $254 \mathrm{~nm}$ & 99,99 & $\pm 0,63$ \\
\hline
\end{tabular}


(variabilité minimale) et de la fidélité intermédiaire (variabilité maximale).

Précisons que la répétabilité intra laboratoire correspond à l'étroitesse de l'accord entre les résultats d'essais obtenus dans un même laboratoire, avec une solution identique soumise à la méthode d'essai par le même opérateur, dans un court intervalle de temps et dans des conditions opératoires constantes. Cependant, l'échantillon de départ devant être homogène afin d'éviter une variabilité induite, il a fallu préparer une solution mère homogène en quantité suffisante à partir de laquelle l'étude de la fidélité a été réalisée.

La condition de fidélité intermédiaire est vérifiée en faisant effectuer les manipulations par trois opérateurs distincts, sur un même appareillage, à des jours différents, aves la même méthode et sur un échantillon initial homogène. Cette étude est réalisée six fois par les trois opérateurs, à un niveau de concentration se situant au milieu du domaine de linéarité (Q3). Les données obtenues pour la cocaïne à $230 \mathrm{~nm}$ sont présentées au tableau VI.

\section{Paramètres statistiques et résultats}

Calcul de l'homogénéité des variances : test de Cochran

Pour les longueurs d'onde testées, quelques valeurs de $\mathrm{C}_{\text {test }}$ sont supérieures à $\mathrm{C}_{\text {aberrant }}$ et un certain nombre d'entre elles se situent entre $\mathrm{C}_{\text {crit }}$ et $\mathrm{C}_{\text {aberrant }}$. Comme précédemment, nous ne garderons que les longueurs d'onde pour lesquelles $\mathrm{C}_{\text {test }}$ est inférieur à $\mathrm{C}_{\text {crit }}$ (seuil de $5 \%$ ). La chloroquine à 210 et à $254 \mathrm{~nm}$ est donc exclue des tests, au même titre que la lidocaïne à $230 \mathrm{~nm}$ et que la benzocaïne à 230 et à $292 \mathrm{~nm}$ (exemple de calcul au tableau VII).

Tableau VII : Test d'homogénéité des variances intragroupes pour la fidélité (test de Cochran) - Cocaïne $230 \mathrm{~nm}$.

\begin{tabular}{|c|c|c|c|}
\hline Groupes (j) & $\mathbf{1}$ & $\mathbf{2}$ & $\mathbf{3}$ \\
\hline $\mathbf{n}_{\mathbf{j}}$ & 6 & 6 & 6 \\
\hline $\mathbf{S}_{\mathbf{j}}$ & 0,1177 & 0,2087 & 0,1739 \\
\hline $\mathbf{m}_{\mathbf{j}}$ & 102,75 & 104,17 & 104,81 \\
\hline
\end{tabular}

$C_{\text {calculé }}=0,2087 \times 0,5003=0,4171<0,707$ (seuil le plus restrictif : $95 \%$ )

Les 3 variances intra groupes peuvent être considérées comme homogènes au risque de $1 \%$

Tableau VI : Fidélité : résultats Cocaïne $230 \mathrm{~nm}$.

\begin{tabular}{|c|c|c|c|c|c|c|c|}
\hline Groupe & Essai & $\begin{array}{c}\text { quantité } \\
\text { introduite }\end{array}$ & $\begin{array}{c}\text { Surface } \\
\text { mol }\end{array}$ & $\begin{array}{c}\text { Surface } \\
\text { Etat Int }\end{array}$ & $\begin{array}{c}\text { Signal } \\
\text { Quantité } \\
\text { retrouvée }\end{array}$ & \% recouvrement \\
\hline \multirow{3}{*}{ 1 } & essai 1/1 & 30 & 2767,6 & 6117,0 & 0,452 & 30,8 & 102,7 \\
& essai 2/1 & 30 & 2779,1 & 6124,5 & 0,454 & 30,9 & 103,0 \\
& essai 3/1 & 30 & 2770,8 & 6119,9 & 0,453 & 30,8 & 102,8 \\
& essai 4/1 & 30 & 2778,3 & 6127,8 & 0,453 & 30,9 & 103,0 \\
& essai 5/1 & 30 & 2751,1 & 6119,9 & 0,450 & 30,6 & 102,1 \\
\hline \multirow{3}{*}{ 2 } & essai 6/1 & 30 & 2771,8 & 6119,1 & 0,453 & 30,9 & 102,9 \\
\hline & essai 1/2 & 30 & 2799,5 & 6130,8 & 0,457 & 31,1 & 103,7 \\
& essai 2/2 & 30 & 2802,1 & 6122,9 & 0,458 & 31,2 & 103,9 \\
& essai 3/2 & 30 & 2808,2 & 6135,8 & 0,458 & 31,2 & 103,9 \\
& essai 4/2 & 30 & 2808,9 & 6130,8 & 0,458 & 31,2 & 104,0 \\
& essai 5/2 & 30 & 2821,4 & 6130,9 & 0,460 & 31,3 & 104,5 \\
\hline \multirow{2}{*}{ 3 } & essai 6/2 & 30 & 2832,2 & 6129,3 & 0,462 & 31,5 & 104,9 \\
\hline & essai 1/3 & 30 & 2817,8 & 6128,2 & 0,460 & 31,3 & 104,4 \\
& essai 2/3 & 30 & 2833,2 & 6136,1 & 0,462 & 31,5 & 104,8 \\
& essai 3/3 & 30 & 2830,7 & 6134,6 & 0,461 & 31,4 & 104,8 \\
& essai 4/3 & 30 & 2820,4 & 6140,6 & 0,459 & 31,3 & 104,3 \\
& essai 5/3 & 30 & 2841,8 & 6138,9 & 0,463 & 31,5 & 105,1 \\
& essai 6/3 & 30 & 2846,9 & 6132,7 & 0,464 & 31,6 & 105,4 \\
\hline
\end{tabular}




\section{Étude des variances}

Les variances prises en compte pour l'étude de la fidélité sont les suivantes :

- Calcul de la variance de répétabilité

Selon la formule suivante : $S_{r}^{2}=\sum_{j=1}^{3} \frac{S_{j}^{2}}{3}$

- Calcul de la variance intergroupe

Selon la formule suivante : $S_{g}^{2}=\frac{\sum_{j=1}^{3}\left(M j-\overline{M)^{2}}\right.}{p-1}-\frac{S_{r}^{2}}{n}$

Avec $\mathrm{M}_{\mathrm{j}}=$ moyenne des groupes

et $\overline{\mathrm{M}}=$ Moyenne des moyennes des groupes

\section{- La variance de reproductibilité $\quad S_{R}^{2}=S_{r}^{2}+S_{g}^{2}$}

A partir de chacune de ces variances, il est possible de calculer un coefficient de variation en divisant la racine carrée de la variance par la moyenne de l'ensemble des valeurs. Ce coefficient de variation est considéré correct en dessous de $10 \%$.

Après étude, toutes les variances peuvent être considérées comme correctes. Les longueurs d'onde analysées sont donc répétables, reproductibles et ont une variance intergroupe satisfaisante. Par exemple, le $\mathrm{CV}_{\mathrm{r}}$ de la cocaïne à $230 \mathrm{~nm}$ est de $0,39 \%$, tandis que son $\mathrm{CV}_{\mathrm{R}}$ est de $1,07 \%$.

L'étude de la fidélité est le dernier test statistique permettant de conclure sur la validité des paramètres choisis. Les substances ayant passé avec succès tous les test de validation, sont la cocaïne et la caféine à 210,230 et $254 \mathrm{~nm}$, la lidocaïne à 210 et $254 \mathrm{~nm}$, le paracétamol à $254 \mathrm{~nm}$, la chloroquine à $230 \mathrm{~nm}$ et la benzocaïne à $210 \mathrm{~nm}$ (toutes les mesures ont été effectuées avec l'étalon interne à $254 \mathrm{~nm}$ ).

\section{Limites de détection et de quantification}

\section{Définitions}

La limite de détection est la plus petite quantité d'un analyte à examiner dans un échantillon, pouvant être détectée et considérée comme différente de la valeur du blanc (avec une probabilité donnée), mais non nécessairement quantifiée.
La limite de quantification, quant à elle, est la plus petite quantité d'un analyte à examiner dans un échantillon pouvant être déterminée quantitativement dans des conditions expérimentales décrites dans la méthode avec une variabilité définie (coefficient de variation déterminé).

\section{Détermination et vérification de la limite de quantification}

La limite de quantification se détermine graphiquement ou par calcul, puis elle se vérifie en surchargeant un blanc matrice avec une quantité choisie d'analyte. En pratique, il s'agit de préparer dix solutions contenant la quantité de substance déterminée et de les analyser selon le protocole validé précédemment. Ensuite, à l'aide de la droite de régression établie lors de l'évaluation de la linéarité et de l'aire du pic obtenue, une concentration $\chi_{\mathrm{LQ}}$ est déterminée puis comparée à la limite de quantification choisie. Grâce aux dix répétitions effectuées, il est possible de calculer la moyenne des valeurs et un écart type afin de vérifier les critères de fidélité et d'exactitude suivants (14) :

- Critère de fidélité :

$$
C V=\frac{S_{L Q}}{L Q}
$$

Si $5 \mathrm{~S}_{\mathrm{LQ}}<\mathrm{LQ}$ alors la valeur 0 n'est pas acceptable comme limite de quantification. Cela revient à vérifier que le coefficient de variation par rapport à LQ est inférieur à $20 \%$.

- Critère d'exactitude :

$$
\frac{\mathrm{LQ}-\overline{\mathrm{x}}_{\mathrm{LQ}}}{\mathrm{s}_{\mathrm{LQ}} / \sqrt{\mathrm{n}}}
$$

avec $x_{L Q}=$ moyenne calculée

et $S_{L Q}=$ écart type

et $L Q=$ limite de quantification choisie

Ce calcul permet de s'assurer que la grandeur moyenne mesurée $\left(\chi_{\mathrm{LQ}}\right)$ n'est pas différente de la limite de quantification choisie. Pour cela il faut s'assurer que la valeur absolue de ce rapport est strictement inférieure à la valeur critique : $10 \%$.

Ces manipulations sont effectuées pour chaque produit de la gamme. Nous présenterons au tableau VIII, uniquement les résultats finaux, c'est à dire les concentrations maximales testées.

\section{Détermination de la limite de détection}

Dans le cas d'une validation complète, la limite de détection est obtenue en divisant par 3 la limite de quantification. Les limites de détection obtenues sont regroupées dans le tableau VIII.

En dessous de ces valeurs, nous ne pourrons pas conclure sur la présence de ces produits dans une poudre. 
Tableau VIII : Limites de quantification et de détection déterminées (en $\mathrm{mg} / \mathrm{l})$.

\begin{tabular}{|l|c|c|}
\hline & Limite de quantification (mg/l) & Limite de détection (mg/l) \\
\hline Paracétamol à $254 \mathrm{~nm}$ & 3 & 1 \\
\hline Caféine à $210 \mathrm{~nm}$ & 2 & 0,67 \\
\hline Caféine à $230,254 \mathrm{~nm}$ & 5 & 1,67 \\
\hline Chloroquine à $230 \mathrm{~nm}$ & 1 & 0,67 \\
\hline Lidocaïne à $210 \mathrm{~nm}$ & 1 & 0,67 \\
\hline Lidocaïne à $254 \mathrm{~nm}$ & 3 & 1 \\
\hline Cocaïne à $230 \mathrm{~nm}$ & 7 & 2,33 \\
\hline Cocaïne à $210,254 \mathrm{~nm}$ & 5 & 1,67 \\
\hline Benzocaïne à $210 \mathrm{~nm}$ & 3 & 1 \\
\hline
\end{tabular}

\section{Conclusion}

L'étude réalisée sur les produits de coupage a montré que la liste établie n'était pas exhaustive. La méthode mise au point permet la quantification des principaux diluants et adultérants couramment rencontrés dans les poudres de cocaïne. Par contre, il n'est pas exclu qu'à long terme de nouveaux produits soient mis en évidence dans la cocaïne de rue. Il faudra alors en tenir compte lors de l'analyse et apporter les modifications nécessaires aussi bien au niveau de la méthode que de la validation.

En ce qui concerne la validation réalisée à partir du guide de validation de la SFSTP, il est préférable que celle-ci permette l'étude des chromatogrammes sur plusieurs longueurs d'onde, afin de conférer une plus grande souplesse à l'analyse.

Le bilan que nous pouvons effectuer sur les huit molécules testées est le suivant :

La procaïne et la benzoylecgonine n'ont pu être validée probablement à cause d'erreurs systématiques. En effet, la benzoylecgonine est un produit pour lequel il est difficile de connaître avec précision le degré d'hydratation.

Le paracétamol, la chloroquine et la benzocaïne ont été validés pour une seule longueur d'onde avec l'étalon interne (Ethylparaben) à $254 \mathrm{~nm}$. Ces conditions de détection seront donc impératives pour le traitement des chromatogrammes.

En revanche, la validation statistique de la caféine, de la lidocaïne et de la cocaïne est tout à fait satisfaisante puisqu'elle offre la possibilité de traiter leurs spectres avec plusieurs longueurs d'onde engendrant ainsi une plus grande souplesse de traitement.

\section{Références}

1. Nations unies, (1986) Méthodes Recommandées pour l'identification de la Cocaïne, New York, 1-9 et 28-9.

2. Pharmeuropa, guide technique, Validation analytique, chapitre III, Novembre 1996, 29.

3. Jamdar S., Pantuck C., Diaz J., Mets B. A rapid, sensitive assay for cocaine and its metabolites in biological fluids using solid-phase extraction and high-performance liquid chromatography. J. Anal. Toxicol. Septembre $2000 ; 24$ : 438-41.

4. Clauwaert K., Van Bocxlaer J., Lambert W., De Leenheer A. Liquid chromatographic determination of cocaine, benzoylecgonine, and cocaethylene in whole blood and serum samples with diode-array detection. J. Chromatogr. Sci. July $1997 ; 35: 321-28$.

5. Caporal-Gautier J., Nivet J.M., Algranti P., Guilloteau M., Histe M., Lallier M., N'Guyen-Huu J.J., Russotto R. Guide de validation analytique : rapport d'une commission SFSTPI. Méthodologie ; STP Pharma pratiques 2 (4) 1992 ; 205-26.

6. International Conference on harmonisation of technical requirements for registration of pharmaceuticals for human use. Validation of analytical procedures : methodology. $1996 ; 2-8$

7. Normalisation française. XP T 90-210. Protocole d'évaluation d'une méthode alternative d'analyse physico-chimique quantitative par rapport à une méthode de référence. AFNOR $1999 ; 1-16$.

8. Validation analytique. Texte CEE référencé III/844/87-FR, projet $n^{\circ} 11$; juillet 1989.

9. Bertrand G., Besacier F., Chaudron H., (2001) Statistiques 2000-2001 Ministère de l'intérieur ; 28-9.

10. Bello P.Y., Toufik A., Gandilhon M. Tendances récentes : Rapport TREND. OFDT, Juin $2001 ; 51$.

11. Spectra analyse (Décembre 2001) $\mathrm{N}^{\circ} 223 ; 14$.

12. Miller J.N., Miller J.C. Statistics of repeated measurements. In : Statistics and chemometrics for analytical chemistry, fourth edition, Harlow, Pearson Education limited, 2000 ; 21-2.

13. Fleury J. Introduction à l'usage des méthodes statistiques en pharmacie. Médecine et Hygiène. Genève 1987 ; 222-37.

14. Normalisation française. XP T 90-210. Protocole d'évaluation d'une méthode alternative d'analyse physico-chimique quantitative par rapport à une méthode de référence. AFNOR $1999 ; 17-8$. 